Sepsis neonatal tardía por Streptococcus gallolyticus subsp. pasteurianus en un hospital de Santa Fe. Presentación de caso clínico

Gonzalez, C.F. • Imhoff, I. • Muñoz Cena, G. • Gomez Colussi, A.F. • Argarañá, M.F. 


\section{SEPSIS NEONATAL TARDÍA POR STREPTOCOCCUS GALLOLYTICUS SUBSP. PASTEURIANUS EN UN HOSPITAL DE SANTA FE. PRESENTACIÓN DE CASO CLÍNICO}

Gonzalez, C.F. ${ }^{1 *}$, Imhoff, I. ${ }^{3}$, Muñoz Cena, G. ${ }^{2}$, Gomez Colussi, A.F. ${ }^{2}$, Argarañá, M.F. ${ }^{2}$

cf-gonzalez@outlook.com

\footnotetext{
${ }^{1}$ Carrera de Especialización en Bacteriología Clínica, Facultad de Bioquímica y Ciencias Biológicas, UNL, Santa Fe, Argentina

${ }^{2}$ Sección Microbiología, División Laboratorio, Hospital J.B. Iturraspe. Santa Fe, Argentina

${ }^{3}$ Centro Perinatológico, División Neonatología, Hospital J.B. Iturraspe, Santa Fe, Argentina
}

\section{RESUMEN}

Streptococcus gallolyticus subsp. pasteurianus es un coco gram positivo, catalasa negativo, perteneciente al grupo Streptococcus bovis. Es un infrecuente agente causal de infección en humanos. Si bien hay pocos casos reportados ha sido una de las subespecies más implicadas en meningitis y sepsis neonatal. Su hallazgo es de importancia debido a la elevada morbimortalidad que presenta en este grupo de pacientes. En este trabajo presentamos un caso de sepsis neonatal tardía con complicación meníngea en un recién nacido pretérmino de un hospital de Santa Fe.

Palabras claves: Estreptococos grupo viridans, meningitis, recién nacido, Streptococcus bovis

\section{SUMMARY}

\section{Late neonatal sepsis due to Streptococcus gallolyticus subsp. pasteurianus in a Santa Fe hospital}

Streptococcus gallolyticus subsp. pasteurianus is a catalase-negative, gram-positive cocci, belonging to the group Streptococcus bovis. It is an uncommon causal agent of infection in humans. Although there are few reported cases it's one of the subspecies most involved in meningitis and neonatal sepsis. Its finding is of importance due to the high morbidity and mortality presented in this group of patients. In this work we present a case of late neonatal sepsis with meningeal complication in a preterm newborn from a hospital in Santa Fe.

Keywords: Viridans group streptococci, meningitis, newborn, Streptococcus bovis 


\section{INTRODUCCIÓN}

La sepsis se define como la disfunción orgánica potencialmente mortal causada por una respuesta desregulada del huésped ante una infección $(1,2)$.

La sepsis neonatal constituye una causa frecuente de mortalidad neonatal y desarrollo de secuelas neurocognitivas $(3,4)$. Puede clasificarse, de acuerdo al tiempo de presentación, en temprana o tardía.

La sepsis neonatal tardía se presenta después de las 72 horas de vida, es más frecuente en recién nacidos prematuros con bajo peso al nacer y hospitalización prolongada en cuidados intensivos. Los factores de riesgo asociados a este tipo de infección son la presencia de lesiones en piel o mucosas en el neonato; la exposición a procedimientos invasivos (catéter venoso central, intubación traqueal, etc.), que funcionan como puerta de entrada de microorganismos y la antibioticoterapia prolongada (3).

El sistema inmunitario inmaduro del neonato pretérmino y su exposición a microorganismos del entorno aumentan la susceptibilidad a una infección invasiva, siendo la contaminación de las manos la fuente más común de infección posnatal (5).

Streptococcus bovis constituye un grupo de cocos gram positivos anaerobios facultativos que expresan el antígeno $\mathrm{D}$ de Lancefield en su superficie. Según sus diferencias bioquímicas se han clasificado en S. bovis biotipo I (fermentación de manitol), S. bovis biotipo II/1 (manitol negativo y $\beta$-glucuronidasa negativa) y biotipo II/2 (manitol negativo y $\beta$-glucuronidasa positiva). Mediante técnicas de biología molecular se ha demostrado que S. bovis biotipo I corresponde a Streptococcus gallolyticus subsp. gallolyticus, que el biotipo II/1 es Streptococcus infantarius subsp. infantarius (renombrado Streptococcus lutetiensis) y el biotipo II/2 se corresponde con S. gallolyticus subsp. pasteurianus (6).

Streptococcus gallolyticus subsp. pasteurianus es parte de la microbiota intestinal humana y puede colonizar de forma asintomática el tracto genitourinario de la mujer embarazada antes del parto. En consecuencia, la transmisión vertical de este microorganismo desde la madre al recién nacido a través del canal de parto o el ambiente posnatal contaminado, podrían ser posibles vías de infección neonatal (7). Las complicaciones más graves son bacteriemia y meningitis neonatal $(8,9)$.

En el tratamiento de infecciones por Streptococcus gallolyticus subsp. pasteurianus, en especial en meningitis, es frecuente el uso de penicilina intravenosa, ampicilina, cefotaxima y ceftriaxona (7).

El objetivo de este trabajo es comunicar el primer caso de sepsis neonatal tardía por Streptococcus gallolyticus subsp. pasteurianus en un hospital de la ciudad de Santa Fe. 


\section{CASO CLÍNICO}

Se presenta el caso de un recién nacido pretérmino de sexo masculino, nacido mediante parto vaginal a las 28 semanas de gestación, con un peso de $1.240 \mathrm{~g}$.

\section{ANTECEDENTES DE LA MADRE}

En el embarazo, la serología fue negativa para virus de hepatitis B y C, virus de la inmunodeficiencia humana, sífilis y chagas; fue positiva para toxoplasmosis (IgG). No se realizó búsqueda de colonización vaginal y anal por Streptococcus agalactiae antes del parto. Los antecedentes de la paciente evidencian dos gestas anteriores, con dos nacidos vivos (uno mediante parto natural y otro mediante cesárea), de los cuales uno fallece en la primera semana de vida y el otro a los 11 meses de vida.

Al momento del parto presentó incompetencia cervical con membranas en reloj de arena y recibió ampicilina. Se enviaron al laboratorio muestras para cultivo de orina, placenta, hisopados anal y vaginal para búsqueda de Streptococcus agalactiae. No se obtuvo desarrollo en ninguna de las muestras procesadas.

El niño nació vigoroso, presentó maduración pulmonar incompleta y respiraciones inefectivas por lo que se intubó, se administró surfactante pulmonar y se internó en unidad neonatal de cuidados intensivos. Ante la sospecha de sepsis recibió ampicilina y gentamicina durante 96 horas, se suspendió debido a la buena evolución clínica y a los cultivos con resultados negativos. Permaneció en asistencia respiratoria mecánica durante 48 horas, se retiró el tubo de manera programada y continuó con ventilación no invasiva mediante presión positiva continua durante 6 días.

A los 22 días de vida presentó mal estado general, al examen físico se lo encontró pálido, hipoactivo, mal perfundido, con respiraciones inefectivas y abdomen distendido, tenso y doloroso, por lo que requirió asistencia respiratoria mecánica. Se comenzó tratamiento empírico con vancomicina y meropenem, se le administraron inotrópicos. Se realizó transfusión de glóbulos rojos y plasma fresco con el fin de aportar factores de coagulación.

Se tomaron muestras de sangre y líquido cefalorraquídeo. El examen citológico y físicoquímico del líquido cefalorraquídeo determinaron la presencia de 1 leucocito/ul; $1,54 \mathrm{~g} / \mathrm{l}$ de proteínas y 0,72 g/l de glucosa. El examen de sangre mostró una leucocitosis moderada de 10.240 leucocitos/ul, glucemia de 0,96 g/l y un estado ácido base alterado.

Se enviaron al laboratorio de microbiología dos frascos de hemocultivos que se incubaron en el equipo automatizado Bact Alert (bioMérieux), y líquido cefalorraquídeo que se sembró en agar sangre, agar chocolate y caldo tioglicolato. De ambas muestras se obtuvo desarrollo de cocos gram positivos identificados como Streptococcus gallolyticus subsp. pasteurianus mediante el sistema automatizado Vitek 2C (bioMérieux, Francia) y pruebas bioquímicas convencionales: catalasa negativo, PYR (producción de pirronidonil arilamidasa) 
negativo, bilis esculina positivo, hidrólisis de Esculina positivo, $\mathrm{NaCl} 6,5 \%$ negativo, manitol negativo, trehalosa positivo. Además, se realizó urocultivo y no se obtuvo desarrollo de microorganismos.

Luego de 24 horas de tratamiento el paciente evolucionó de manera favorable. Se retiró el tubo y se suspendió la administración de inotrópicos. Se administraron 7 días de meropenem y 21 días de vancomicina, con hemocultivos de control negativos. Se observó buena evolución clínica, con ecografías transfontanelares sin alteraciones. Se otorgó alta médica a las 36 semanas de edad gestacional, cuando alcanzó el peso deseado (2.200 g), toleró lactancia materna y alimentación enteral, presentó estabilidad y suficiencia respiratoria.

\section{DISCUSIÓN}

Streptococcus gallolyticus subsp. pasteurianus es un agente etiológico muy infrecuente de sepsis neonatal (10).

El paciente presentó una sepsis neonatal tardía debido a que la sintomatología se presentó a los 22 días de vida. En este caso, la enfermedad invasora se confirmó con el aislamiento del microorganismo en líquido cefalorraquídeo y hemocultivos. La inmadurez del sistema inmunitario del neonato constituyó el principal factor de riesgo para adquirir la infección. En nuestro caso, la vía de transmisión de Streptococcus gallolyticus subsp. pasteurianus es incierta, sin embargo, debido a la presentación tardía de los síntomas podemos sugerir que el patógeno se encontraba en el entorno posnatal.

Se destaca la importancia de identificación del grupo Streptococcus bovis hasta el nivel de subespecie, debido a que cada una de las subespecies de este grupo tienen distinta implicancia clínica. Aislamientos de Streptococcus gallolyticus subsp. gallolyticus se han asociado a endocarditis y cáncer de colon en adultos; Streptococcus infantarius subsp. infantarius se han asociado a neoplasias no colónicas en adultos y aislamientos de Streptococcus gallolyticus subsp. pasteurianus se han encontrado en adultos y neonatos con cuadros de sepsis y meningitis (11).

El pronóstico de sepsis neonatal suele ser bueno con tratamiento antibiótico instaurado de forma precoz, la comunicación inmediata al médico tratante del microorganismo correctamente identificado, son relevantes para realizar un tratamiento adecuado. Se utilizó, desde el inicio, vancomicina y meropenem como tratamiento empírico y debido a la buena evolución del paciente se completó el esquema con vancomicina hasta alta médica.

\section{Conflicto de intereses}

Los autores declaran no tener ningún conflicto de intereses.

La presente investigación no ha recibido ninguna beca específica de agencias de los sectores público, comercial, o sin ánimo de lucro. 


\section{BIBLIOGRAFÍA}

1-Singer, M.; Deutschman, C.S.; Seymour, C.W.; Shankar-Hari, M.; Annane, D.; Bauer, M.; Bellomo, R.; Bernard, G.R.; Chiche, J.D.; Coopersmith, C.M.; Hotchkiss, R.S.; Levy, M.M.; Marshall, J.C.; Martin, G.S.; Opal, S.M.; Rubenfeld, G.D.; Van der Poll, T.; Vincent, J.L. and Angus, D.C, 2016. The third international consensus definitions for sepsis and septic shock. JAMA. 315, 8: 801-810.

2-Verdonk, F.; Mazeraud, A.; Chrétien, F.; Sharshar, T., 2020. Sepsis-Associated Encephalopathy. En: Chrétien F.; Thong Wong, K.; Sharer, L.R.; Keohane, C. and Gray, F. (Ed.), Infections of the Central Nervous System: Pathology and Genetics, John Wiley \& Sons Ltd (Francia), I. 2: 11-20.

3-Hentges, C.R.; Silveira, R.C.; Procianoy, R.C.; Carvalho, C.G.; Filipouski, G.R.; Fuentefria, R.N.; Marquezotti, F.; Terrazan, A.C, 2014. Association of late-onset neonatal sepsis with late neurodevelopment in the first two years of life of preterm infants with very low birth weight. J ped (Rio J) 90, 1: 50-7.

4-Liang, L.D.; Kotadia, N.; English, L.; Kissoon, N.; Ansermino, J.M.; Kabakyenga, J.; Lavole, P.M. and Wiens, M.O, 2018. Predictors of mortality in neonates and infants hospitalized with sepsis or serious infections in developing countries: a sistematic review. Front in ped 6, 277: 1-12.

5-Shane, A.L.; Sánchez, P.J.; Stoll, B.J., 2017. Neonatal sepsis. Lancet. 390, 10104: 1770-1780.

6-Romero Hernández, B.; Del Campo, R.; Cantón, R., 2013. Streptococcus bovis, situación taxonómica, relevancia clínica y sensibilidad antimicrobiana. Enf Inf y mic clín. 31, 1: 14 1-9.

7-Li, Y.; Chen, X.; Zhang, Z.; Wang, L.; Wang, J.; Zeng, J.; Yang, J.; Lu, B, 2019. Microbiological and clinical characteristics of Streptococcus gallolyticus subsp. pasteurianus infection in China. BMC Inf Dis 19, 791: 111.

8-Binghuai, L.; Wenjun, S.; Xinxin, L, 2013. Intrauterine infection and post-partum bacteraemia due to Streptococcus gallolyticus subsp. pasteurianus. J Med Microbiology 62, 10: 1617-1619.

9-Nguyen, M.; Idriss, S.; Guzman, E.; De Oliveira, E., 2019. Neonatal meningitis, endocarditis, and pneumonitis due to Streptococcus gallolyticus subsp. pasteurianus: a case report. BMC Ped. 19, 265; 1-5.

10-Floret, N.; Bailly, P.; Thouverez, M.; Blanchot, C.; Alez-Martin, D.; Menget, A.; Thiriez, G.; Hoen, B.; Talon, D.; Bertrand, X, 2010. A cluster of bloodstream infections caused by Streptococcus gallolyticus subsp. pasteurianus that involved 5 preterm neonates in a University Hospital during a 2-month period. Inf Cont and Hosp Epidemiology 31, 2: 194-196.

11-Lopardo, H.A., 2016. Cocos gram positivos catalasa negativos. En: Lopardo, H.A.; Predari, S.C.; Vay, C. (Ed.), Manual de microbiología clínica de la asociación argentina de microbiología. Asociación argentina de microbiología (Argentina), I. 148-150. 